\title{
Surgical fixation of posterior cruciate ligament avulsion fracture from tibial attachment using cannulated cancellous screw: a clinical, radiological and functional outcome evaluation
}

\author{
Aniruddha Mondal ${ }^{1}$, Ayon Das ${ }^{2 *}$ \\ ${ }^{1}$ Department of Orthopaedics, NRS Medical College and Hospital, Kolkata, West Bengal, India \\ ${ }^{2}$ Department of Orthopaedics, IPGMER and SSKM Hospital, Kolkata, West Bengal, India \\ Received: 01 May 2021 \\ Revised: 11 May 2021 \\ Accepted: 12 May 2021 \\ *Correspondence: \\ Dr. Ayon Das, \\ E-mail: dr.ayondas@gmail.com \\ Copyright: $\odot$ the author(s), publisher and licensee Medip Academy. This is an open-access article distributed under \\ the terms of the Creative Commons Attribution Non-Commercial License, which permits unrestricted non-commercial \\ use, distribution, and reproduction in any medium, provided the original work is properly cited.
}

\begin{abstract}
Background: PCL (posterior cruciate ligament) is the main posterior stabilizer of the knee and plays a role in central axis controlling and imparting rotational stability to the knee. Injury to PCL presents commonly with avulsion fractures from its tibial attachment. An avulsion fracture of the PCL, if not surgically fixed, may lead to secondary changes in the knee joint. The aim of the study was to evaluate the clinical and functional results in patients with PCL tibial avulsion fractures treated by CC (cannulated cancellous) screw fixation.

Methods: This was a prospective study conducted at a tertiary care government hospital in Kolkata, between December 2018 to July 2020 on patients who underwent CC screw fixation for post-traumatic PCL avulsion fracture from tibial site. All patients were followed up for 9 months. Lysholm knee score was used to assess the functional outcomes of the patients.

Results: In a total of 10 patients, 9 (90\%) patients showed excellent and $1(10 \%)$ patient had good result during the final follow-up. $80 \%$ patients did not develop any complications. At the end of 9 months, the mean Lysholm score was 95.6. $8(80 \%)$ patients achieved almost full knee ROM post-operatively by the end of final follow-up.

Conclusions: Surgical fixation using CC screws is a simple, easy, safe and reproducible method without requiring significant expertise for achieving good stability in PCL tibial site avulsion fractures, where early intervention prevents significant late disability as it provides an excellent clinical, functional and radiographic outcome along with good joint function.
\end{abstract}

Keywords: Posterior cruciate ligament, Avulsion fracture, CC screw, Fracture fixation, Lysholm score

\section{INTRODUCTION}

PCL is the stronger of the two cruciate ligaments of knee and has a major role in stabilizing the knee joint. ${ }^{1,2}$ It acts as a primary restraint against posterior tibial displacement and in concert with the anterior cruciate ligament (ACL), to regulate external rotation of the knee during extension. ${ }^{3}$
PCL injuries are estimated to account for $20 \%$ of knee ligament injuries. This incidence is higher especially in cases resulting from high-energy trauma, such in motorcycle and car accidents with dashboard injuries, while, in an athletic population, this injury is more closely associated to contact sports. ${ }^{4,5}$

The most commonly reported mechanism of isolated PCL injury is a posteriorly directed blow to the anterior aspect 
of the proximal tibia with the knee flexed at $90^{\circ}{ }^{6}$ Another mechanism is sudden hyperextension associated with varus or valgus force or hyperflexion of the knee. ${ }^{7}$

A PCL disruption may occur as avulsion at the femoral origin or tibial insertion or as a midsubstance tear. ${ }^{8-10}$ Avulsion of the tibial insertion of the PCL is believed to be an uncommon injury. A nondisplaced bony avulsion can be treated conservatively with a plaster cast, holding the knee flexed and the tibia pulled forward to diminish tension on the PCL.

Many studies have demonstrated that the chronic PCL insufficiencies may result in degenerative arthritis of medial and patellofemoral compartments and increased risk of meniscal tear. There is general consensus that a displaced bony PCL avulsion should be surgically reduced and fixed immediately to stabilize the knee joint and avoid the morbidities associated with non-union such as delayed osteoarthritis and permanent knee pain. ${ }^{11-13}$

Repair of avulsion injuries can be done with an open or arthroscopic technique. Biomechanical studies have also demonstrated comparable results of screw fixation using open or arthroscopic means. ${ }^{14}$ However, arthroscopic repair is expensive, technically more challenging, requires specialized equipment, has a steep learning curve and the fracture fixation is potentially unstable. ${ }^{15,16}$ Open reduction and internal fixation using screws has been considered a favourable method to manage PCL avulsion injuries producing satisfactory results.

There is currently a wide variety of materials available for internal fixation, including lag screws, steel wires, absorbable screws, suture anchors, spring plate and straddle nails. ${ }^{17,18}$ Fixation with screws has shown favourable results. However, no one technique has been considered a gold standard for avulsion fractures of the PCL.

The objective of this study was to represent our experience comprising cases of PCL avulsion fracture from its tibial site of attachment which were treated by open reduction and internal fixation using $\mathrm{CC}$ screw to see whether the technique achieves stable knees, good range of motion, acceptable radiological union with improved clinical and functional outcome using this easily available implant via a simple and safe surgical technique.

\section{METHODS}

This was a prospective study conducted in accordance with the ethical standards of the institutional review board. 10 patients with post-traumatic PCL tibial site avulsion fracture who were treated by $\mathrm{CC}$ screw fixation in the Department of Orthopaedics, NRS Medical College and Hospital, Kolkata from December 2018 to July 2020 and fulfilling the inclusion criteria were considered in this study.

\section{Inclusion criteria}

Skeletally mature patients above 18 years of age, patients with isolated tibial avulsion fracture of PCL, fracture displacement $>3 \mathrm{~mm}$, avulsed fracture fragment size $>20$ $\mathrm{mm}$ and fresh injury ( $<3$ weeks old) were included in the study.

\section{Exclusion criteria}

Patients with associated other ligament or meniscus injury of same joint, occult midsubstance injury of the avulsed PCL or any other bone fracture of ipsilateral lower limb, previous ipsilateral knee surgery and patients with polytrauma or head injuries that definitely influence rehabilitation were excluded from the study.

\section{Operative procedure}

The patients were subjected to a thorough history, clinical examination and pre-operative routine laboratory investigations, which were supplemented by radiographs in antero-posterior and lateral view of the knee joint along with a CT scan with 3D reconstruction and an MRI.

All the patients were operated under spinal anaesthesia. Patients were positioned prone on a radiolucent operating table with proper padding of the pressure areas and the lower extremity was held in $30^{\circ}$ flexion at the knee joint over a bolster at ankle. Pneumatic tourniquet was used in all cases.

After proper aseptic dressing and draping, an inverted Lshaped incision was made over the posteromedial corner of the knee joint with the horizontal end near the flexion crease of the knee and the vertical limb overlying the medial aspect of the gastrocnemius muscle. The deep fascia was carefully divided in the midline and the short saphenous vein and sural nerve were identified and protected. The interval between medial head of gastrocnemius and semimembranosus tendon was identified and dissection was carried out bluntly until the posterior capsule of knee joint was reached. Then the joint capsule was incised longitudinally to expose the PCL and avulsed fracture fragment, after protecting and retracting the neurovascular bundles laterally. The avulsed fracture fragment was debrided and reduced in slight flexed position of the knee. The bony fragment was pushed down and secured with a guide wire and the position was verified under a fluoroscope. The bony fragment was then fixed using a $4 \mathrm{~mm}$ partially threaded cannulated cancellous screw with washer. The position of bony fragment was again assessed under fluoroscope and if found adequate, surgical wound was thoroughly washed and then closed in layers. Sterile dressings were applied to the surgical incision and the knee was immobilized in a hinged knee brace.

Postoperatively, the patient was encouraged to do static quadriceps exercises, straight leg raise, ankle pumps from 
second day of surgery. All patients were kept non weight bearing for 6 weeks in a hinged knee brace. Passive knee bending was started after suture removal i.e. after 2 weeks and active knee mobilization was started 3 weeks postsurgery. Partial weight bearing using bilateral axillary crutches was allowed after 6 weeks and full weight bearing after 12 weeks when the knee brace was also discarded. Majority of patients returned to their previous occupation by 6 months whereas heavy activities such as running and sports were allowed only after 9 months.

The patients were regularly followed up for 9 months at 2 weeks, 6 weeks, 12 weeks, 6 months and 9 months interval. Except for the first visit, in which only range of motion and local wound condition was addressed, subsequent visits included thorough clinical and radiological assessment. Clinical examination included posterior drawer test and radiological assessment was done with antero-posterior and lateral radiographs of knee.

\section{Statistical analysis}

The data was collected in microsoft excel (windows 10, version 2016) and statistical software SPSS version 20 was used for analysis. Procedure of the data analysis was transcription, preliminary data inspection, content analysis and interpretation. The categorical variables like age, sex, side, mode of injury were expressed as number of patients and to differentiate using the mean scores a non-parametric Wilcoxon matched paired test was used at 0.05 level of significance.

\section{RESULTS}

\section{Age distribution}

Overall, $90 \%$ patients were between the age group of 20 40 years. The mean age in this study was 32.7 years. The youngest patient was 25 years old and the eldest patient was 42 years old. It might be because younger peoples are more active and involved in outdoor and sports activities which makes them more prone to injuries (Table 1).

\section{Sex distribution}

In this study, 9 patients (90\%) were male and 1 patient was female (10\%). Majority of patients were male which may be because of more outdoor and sports related activities makes them more vulnerable to accidents and trauma.

\section{Side of injury distribution}

In this study, number of right knee involvement was 6 (60\%) which was almost comparable to left knee involvement which was $4(40 \%)$.

Table 1: Age distribution.

\begin{tabular}{|c|c|c|c|}
\hline Age (in years) & Number of patients & Percentage $(\%)$ & P value \\
\hline $20-30$ & 3 & 30.0 & \multirow{4}{*}{0.001} \\
\hline $31-40$ & 6 & 60.0 & \\
\hline$>40$ & 1 & 10.0 & \\
\hline Total & 10 & 100.0 & \\
\hline
\end{tabular}

Table 2: Posterior drawer test distribution.

\begin{tabular}{|lll|}
\hline Laxity (in mm) & $\begin{array}{l}\text { Pre-operative } \\
\text { Number of patients (\%) }\end{array}$ & $\begin{array}{l}\text { Post-operative } \\
\text { Number of patients }(\%)\end{array}$ \\
\hline Grade $\mathbf{~ ( n i l ) ~}$ & 0 & $8(80)$ \\
\hline Grade I (0-5) (mild) & 0 & $2(20)$ \\
\hline Grade II (6-10) (moderate) & $1(10)$ & 0 \\
\hline Grade III (>10) (severe) & $9(90)$ & 0 \\
\hline Total & $10(100)$ & $10(100)$ \\
\hline
\end{tabular}

Table 3: Post-operative final range of motion distribution.

\begin{tabular}{|c|c|c|c|}
\hline $\begin{array}{l}\text { Final ROM } \\
\text { (in degrees) }\end{array}$ & Number of patients & Percentage (\%) & P value \\
\hline 0-120 & 0 & 0 & \multirow{4}{*}{0.001} \\
\hline 0-130 & 2 & 20.0 & \\
\hline $0-140$ & 8 & 80.0 & \\
\hline Total & 10 & 100.0 & \\
\hline
\end{tabular}




\section{Mode of injury distribution}

In this study, $6(60 \%)$ patients were involved in motor vehicle accident, 2 (20\%) suffered a fall and $2(20 \%)$ sustained trauma during sports activities. The most common mode of trauma in case of motor vehicle accident was a dashboard injury (Figure 1).

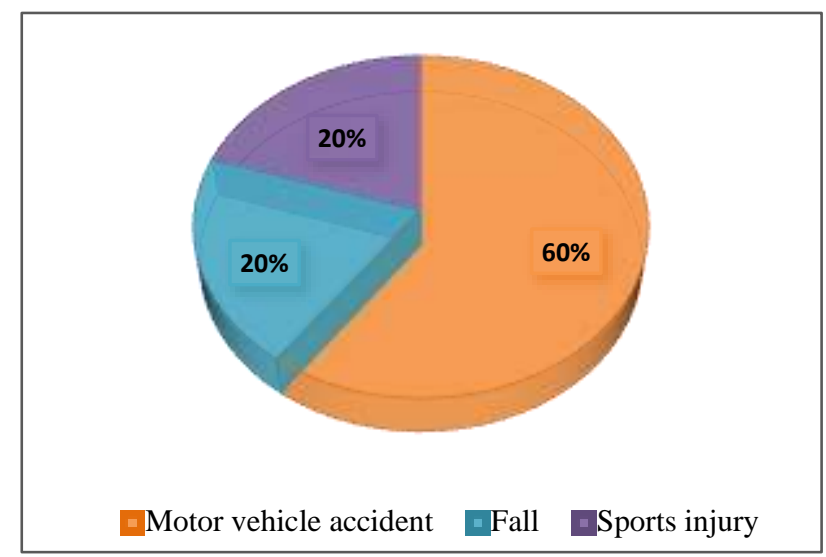

Figure 1: Mode of injury distribution.

\section{Posterior drawer test distribution}

Among 10 patients, majority (90\%) of patients had severe instability at the time of presentation and none of them had nil instability pre-operatively. Post-operatively, $80 \%$ of the patients did not have any instability. There were only 2 cases of mild laxity on clinical examination but subjectively, the patients were not aware of the instability (Table 2).
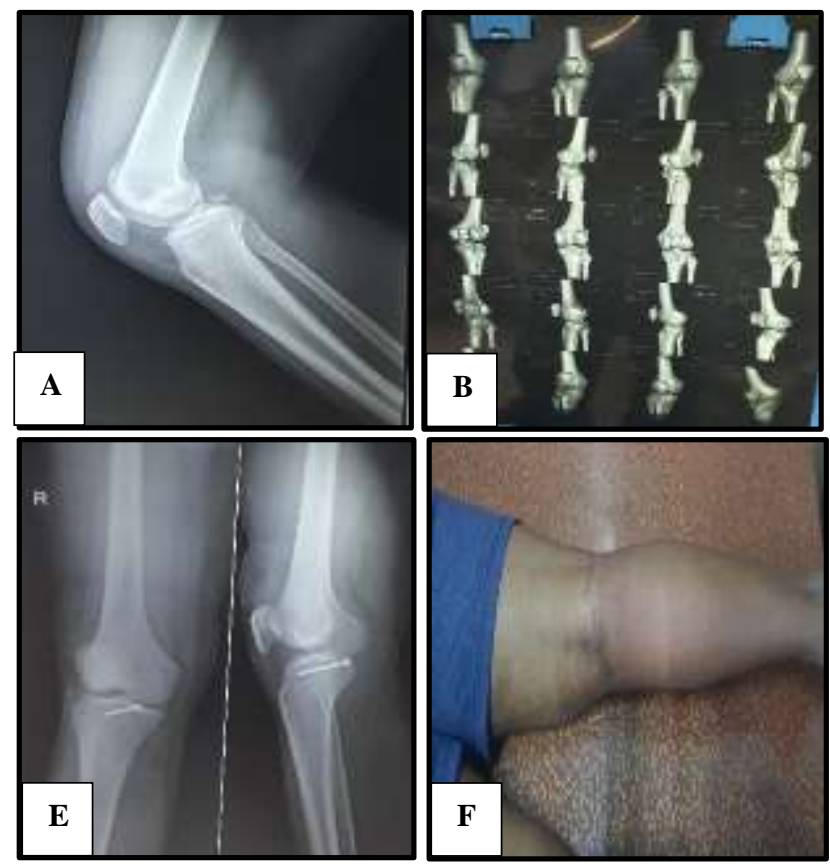

\section{Post-operative final range of motion distribution}

Out of 10 patients, 2 patients had $0-130^{\circ}$ ROM and 8 (80\%) patient had $0-140^{\circ}$ ROM post-operatively by the end of 9 months (Table 3).

\section{Lysholm score}

At the end of final follow-up, the average Lysholm score for 10 patients was 95.6 .

\section{Lysholm score outcome}

In a total of 10 patients, $9(90 \%)$ patients showed excellent and $1(10 \%)$ patient had good result. None of the patients demonstrated fair or poor result post-operatively at the end of 9 months follow-up (Figure 2).

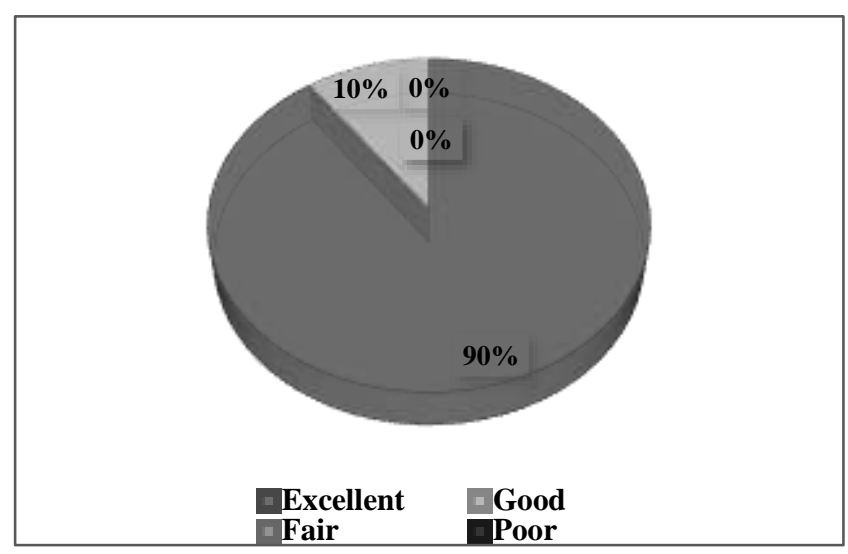

Figure 2: Lysholm score outcome.

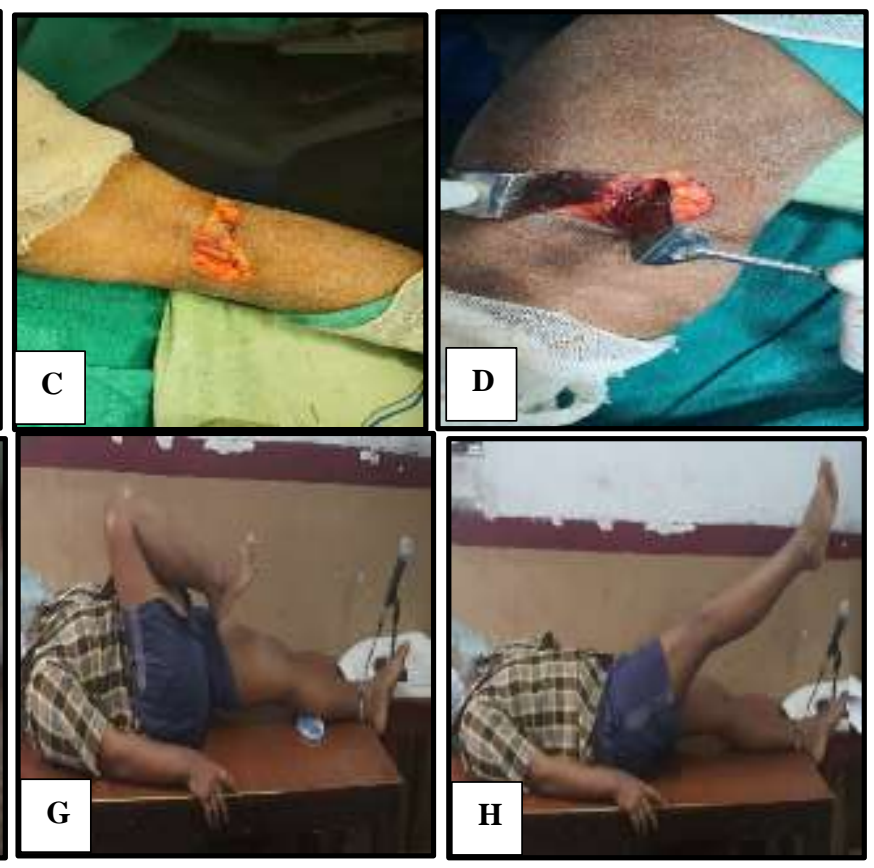

Figure 3: (A) Pre-op X-ray showing PCL avulsion fracture; (B) Pre-op CT scan with 3D reconstruction; (C) Inverted L-shaped surgical incision; (D) Intra-op view of CC screw fixation; (E) Post-op X-ray; (F) Healed surgical scar at 9 months; ( $G$ and $H$ ) Knee ROM at final follow-up. 


\section{Complications}

Among 10 patients, $8(80 \%)$ patients did not have any complications. 1 patient complained of residual intermittent pain during vigorous sports activities. 1 patient had developed a mild wound gaping with superficial infection which subsided with a course of oral antibiotic along with regular sterile and aseptic dressing for 2 weeks.

None of the patients had flexion deformity or extensor lag at final follow-up. Other complications such as non-union, instability or arthrofibrosis were not seen. No patient had to undergo implant removal for hardware related problems.

Majority of patients (90\%), presented within 1 week of injury. All patients regained $90^{\circ}$ of knee motion within 6 weeks of surgery. The average time to radiological union was observed to be around 12 weeks. No intraoperative complications such as excessive bleeding, iatrogenic fracture or neurovascular damage were encountered during the surgical procedure.

\section{DISCUSSION}

The function of PCL is primary restraint for posterior tibial translation during knee flexion and thus stabilization of the knee joint. ${ }^{19}$ Injury to it results in posterior subluxation of the tibia due to instability with increased stress over patellofemoral joint leading to long term disability in the form of chronic pain and cartilage degeneration. ${ }^{20,21}$

Although early diagnosis of PCL tears is difficult and the treatment is controversial, diagnosis and treatment protocol of bony PCL avulsion injuries are universally standard. ${ }^{9,20}$ Regarding the treatment of the PCL tibial avulsion, non-operative treatment is a choice, but loss of ROM of the knee with some residual PCL laxity can be a significant problem in the long term. ${ }^{22}$ Furthermore, osteonecrosis or non-union of the fragment of the tibial insertion of the PCL is another concern because a part of its base is framed by the fragment of the lateral tibial condyle, this is disadvantageous to blood supply. ${ }^{23}$

Although the necessity of surgical treatment of isolated PCL injuries is still controversial, fixation methods for avulsion fractures of the PCL at the tibial insertion have been suggested in different studies. Surgical approaches to the avulsed PCL may be open or arthroscopic, but safety and simplicity of the approach remains a major concern.

Piedade et al evaluated 21 patients with an average age of 30 years, who underwent operative management for the PCL fracture avulsion. Surgical treatment consisted of posterior approach to the knee and fixation of the bone fragment with a screw and washer in 18 cases and transbone ties in 3 cases, where the bone fragment was very small. They assessed the patients objectively (posterior drawer test) and subjectively (Lysholm scale), after a minimum postoperative follow-up of 12 months and found excellent results in $53 \%$ and good in $47 \%$ cases. $^{24}$

Attia et al designed a prospective study to evaluate the efficacy of the treatment of PCL avulsion fractures by fixation using the posteromedial approach. Navicular screw fixation was used in avulsed bony fragments, with washers in 8 patients and without washers in 4 patients. All the avulsion fractures achieved union at an average of 1014 weeks. After union of the fracture, none suffered severe instability. Patients were followed up for an average of 7.58 months. Of the 12 patients included in this study, 4 (33.3\%) showed excellent results, 7 (58.3\%) showed good results and $1(8.3 \%)$ showed a fair result as per Lysholm knee score. ${ }^{25}$

Khatri et al conducted a prospective study on 27 patients with mean follow-up duration of $22.30 \pm 6.82$ months for PCL tibial avulsion fractures treated with open reduction and internal fixation via Burks and Schaffer approach. The mean Lysholm scores at the time of last follow-up was $90.85 \pm 5.58$. The IKDC grades achieved were normal in 20 patients, near normal in 5 and abnormal in 2. The PCL laxity was grade I in 20 cases and grade II in 7 cases. All patients had achieved bony union of tibial avulsion fractures at the time of last follow-up. ${ }^{2}$

Joshi et al performed open reduction and internal fixation using CC screws in 14 patients (mean age, 33.9 years) with isolated PCL avulsion injuries. The minimum follow-up period was 12 months. At the final follow-up, all patients achieved fracture union. The average flexion was $121.7^{\circ} \pm 9.2^{\circ}$ with full extension achieved in all patients. Mild instability (1+) was noted in 4 patients. The Lysholm functional score was excellent in 11 patients, good in 2 patients and fair in 1 patient with an average score of $97 \pm 7.6 .^{26}$

Eladawy et al did a prospective study on 11 patients with PCL avulsion fracture of the tibial attachment who were treated surgically through a posterior approach. The mean age of patients was 24.72 years. Fixation with a single screw and washer was used in 9 cases and multiple screws in 2 cases. All patients were followed for an average of 22 months. All 1 year post-operative radiographs demonstrated union at the fracture site. At the end of the follow-up period, the mean Lysholm score was 89.7 points. 6 patients showed good to excellent outcomes, whereas 5 patients had fair to good outcomes and 1 patient showed a fair outcome. All the patients were satisfied with the results. ${ }^{27}$

Jha et al enrolled 19 cases of isolated posterior cruciate ligament avulsion injury with mean age of $33.21 \pm 9.07$ years. All cases were treated by open reduction and internal fixation through modified posterior approach. The mean duration of follow up was $14 \pm 1.85$ months. All patients achieved union at 3 months. At final follow-up, the mean range of motion was $125.42^{\circ} \pm 6.37^{\circ}$ without any extensor lag. The functional outcome assessed by Lysholm 
scoring system was excellent in 15 cases and good in 4 cases. The mean Lysholm score was 95.26 \pm 5.44 . At final follow-up, posterior drawer test showed grade I instability in 3 patients and remaining 16 patients did not have any instability. ${ }^{28}$

Several techniques and implants have been used for reduction and fixation, according to the size and the degree of comminution of the avulsed fragment and according to the expertise of the treating surgeon. However, nowadays screw fixation has been the most common method used and has shown favorable results.

Limitations of our study included single institution bias, small group of patients, short follow-up period and a lack of control group. Additional prospective and biomechanics studies should be conducted to confirm these outcomes in the future. A multicentre study with more patients is essential to substantiate benefits of this treatment method.

\section{CONCLUSION}

Surgical fixation of PCL avulsion fracture from its tibial attachment using CC screw gives excellent results after stable fixation. It has now become standard of care due to its simple, safe, easy and adequate exposure techniques with minimum soft tissue dissection. It can be done without significant expertise and has a short learning curve. This technique is associated with satisfactory fixation, minimal complications and good recovery of joint function. Time and again it has produced good clinical, functional and radiological outcomes without any longterm disability.

A larger well-designed study is required to be conducted across the country to corroborate the findings of our study.

Funding: No funding sources

Conflict of interest: None declared

Ethical approval: The study was approved by the institutional ethics committee

\section{REFERENCES}

1. Rezazadeh S, Solooki S, Aboulhasani S, Vosoughi AR. Midterm results of open reduction and internal fixation of isolated posterior cruciate ligament avulsion fracture. Eur Orthopaed Traumatol. 2011;1(6):191-5.

2. Khatri K, Sharma V, Lakhotia D, Bhalla R, Farooque K. Posterior cruciate ligament tibial avulsion treated with open reduction and internal fixation through the Burks and Schaffer approach. Malaysian Orthopaed J. 2015;9(2):2.

3. Detenbeck LC. Function of the cruciate ligaments in knee stability. J Sport Med. 1974;2(4):217-21.

4. Wind WM, Bergefeld JA, Parker RD. Evaluation and treatment of posterior cruciate injuries. Am J Sports Med. 2004;32(7):1765-75.
5. Deeham DJ, Pinczewski LA. Arthroscopic reattachment for an avulsion fracture of the tibial insertion of the posterior cruciate ligament. Arthroscopy. 2001;17(4):422-5.

6. Veselko M, Saciri V. Posterior approach for arthroscopic reduction and antegrade fixation of avulsion fracture of the posterior cruciate ligament from the tibia with cannulated screw and washer. Arthroscopy. 2003;19(4):916-21.

7. Fowler PJ, Messieh SS. Isolated posterior cruciate ligament injuries in athletes. Am J Sports Med. 1987;15(6):553-7.

8. Loos WC, Fox JM, Blazina ME, Pizzo WD, Friedman MJ. Acute posterior cruciate ligament injuries. Am J Sports Med. 1981;9(2):86-92.

9. Torisu TA. Isolated avulsion fracture of the tibial attachment of the posterior cruciate ligament. J Bone Joint Surg Am. 1977;59(1):68-72.

10. Jakob RP, Ruegsegger M. Therapy of posterior and posterolateral knee instability. Orthopade. 1993;22(6):401-13.

11. Meyers MH. Isolated avulsion fractures of the tibial attachment of the posterior cruciate ligament of the knee. J Bone Joint Surg. 1975;57(5):669-72.

12. Hooper PO, Silko C, Malcolm TL, Farrow LD. Management of posterior cruciate ligament tibial avulsion injuries: a systematic review. Ame J Sport Med. 2018;46(3):734-42.

13. Katsman A, Strauss EJ, Campbell KA, Alaia MJ. Posterior cruciate ligament avulsion fractures. Curr Rev Musculosk Med. 2018;11(3):503-9.

14. Sasaki SU, Albuquerque RFM, Amatuzzi MM, Pereira CAM. Open screw fixation versus arthroscopic suture fixation of tibial posterior cruciate ligament avulsion injuries: a mechanical comparison. Arthroscopy. 2007;23(11):1226-30.

15. Sabat D, Jain A, Kumar V. Displaced posterior cruciate ligament avulsion fractures: a retrospective comparative study between open posterior approach and arthroscopic single-tunnel suture fixation. Arthroscopy: Journal Arthroscop Rel Surg. 2016;32(1):44-53.

16. Pardiwala DN, Agrawal D, Patil V, Saini U, Dhawal P. Paper 133: comparison of open versus arthroscopic fixation for isolated PCL tibial bony avulsions: a prospective randomized study with minimum 2 year follow-up. Arthroscopy. 2012;28(9):413-4.

17. Sun HQ, Ren YJ, Zhang YQ. Treatment of posterior cruciate ligament avulsion fracture with pressurized staples. Chi J Orthop Trauma. 2011;13:1189-90.

18. Fu YP, Hang CM, Fam HQ. Treatment of posterior cruciate ligament avulsion fracture using anchor system combined with cannulated screw. J Pract Orthop. 2011;17:73-4.

19. Kannus P, Bergfeld J, Jarvinen M, Johnson RJ, Pope $\mathrm{M}$, Renstrom $\mathrm{P}$, et al. Injuries to the Posterior cruciate ligament of the knee. Sports Med. 1991;12(2):11031. 
20. Trickey EL. Rupture of the posterior cruciate ligament of the knee. Journal Bone Joint Surg. Brit Vol. 1968;50(2):334-41.

21. Sonin AH, Fitzgerald SW, Friedman H. Posterior cruciate ligament injury: MR imaging diagnosis and patterns of injury. Radiol. 1994;190(2):455-8.

22. Shelbourne KD, Davis TJ, Patel DV. The natural history of acute, isolated, nonoperatively treated posterior cruciate ligament injuries. Am Journal Sport Med. 1999;27(3):276-83.

23. Ogawa H, Sumi H, Shimizu K. Posterior cruciate ligament mediated avulsion fracture of the lateral tibial condyle: a case report. J Orthopaed Surgery Res. 2010;5(1):1-6.

24. Piedade SR, Mischan MM. Surgical treatment of avulsion fractures of the knee PCL tibial insertion: experience with 21 cases. Acta Ortopédica Brasileira. 2007;15(5):272-5.

25. Attia ME, Zanfaly AI. Fixation of tibial bony avulsion of the posterior cruciate ligament using the posteromedial approach. Egypt Orthopaed J. 2014;49(2):81.
26. Joshi S, Bhatia C, Gondane A, Rai A, Singh S, Gupta $\mathrm{S}$. Open reduction and internal fixation of isolated posterior cruciate ligament avulsion fractures: clinical and functional outcome. Knee Surg Rel Res. 2017;29(3):210.

27. Eladawy A, Abdeen M. Outcomes of surgical treatment of posterior cruciate ligament tibial avulsion fractures through an open posterior approach. Egypt Orthopaed J. 2018;53(3):230.

28. Jha RK, Thapa S. Treatment of tibial avulsion fracture of posterior cruciate ligament by open reduction and internal fixation. $\mathbf{J}$ Nobel Med Coll. 2020;9(1):17-21.

Cite this article as: Mondal A, Das A. Surgical fixation of posterior cruciate ligament avulsion fracture from tibial attachment using cannulated cancellous screw: a clinical, radiological and functional outcome evaluation. Int J Res Orthop 2021;7:720-6. 